\title{
Quasi steady-state models for long-term voltage and frequency dynamics simulation
}

\author{
Marie-Eve Grenier
}

\author{
Daniel Lefebvre
}

\author{
Thierry Van Cutsem, Fellow, IEEE
}

\begin{abstract}
The Quasi Steady-State (QSS) approximation of long-term dynamics relies on time-scale decomposition and consists of replacing faster phenomena by their equilibrium conditions, in order to reduce the complexity of the whole model and increase the computation efficiency of time simulations. This paper describes the extension of a QSS model extensively used in long-term voltage stability studies, to readily incorporate the frequency dynamics that takes place over the same time scale. This extended QSS model relies on a common-frequency assumption. Its advantages, limitations and possible improvements are discussed through simulation results on the Hydro-Québec system, where it has been compared to full time scale simulations. Disturbances with an impact on either frequency or voltages are considered and the coupling between these two aspects of longterm dynamics is briefly discussed.
\end{abstract}

Index Terms-long-term dynamics, frequency dynamics, voltage dynamics, quasi steady-state approximation, governor and turbine modelling

\section{INTRODUCTION}

The QSS approximation of long-term dynamics is a wellknown and proven technique for the fast simulation of power system dynamics. Simply stated, this approximation relies on time-scale decomposition: faster phenomena are represented by their equilibrium conditions instead of their full dynamics [1]. This greatly reduces the complexity of the resulting model and hence provides the computational efficiency required for fast applications such as real-time contingency analysis [2] or training simulators [3].

In stability studies, the general dynamic model of a power system takes on the form:

$$
\begin{aligned}
\mathbf{0} & =\mathbf{g}(\mathbf{x}, \mathbf{y}, \mathbf{z}) \\
\dot{\mathbf{x}} & =\mathbf{f}(\mathbf{x}, \mathbf{y}, \mathbf{z}) \\
\mathbf{z}\left(t_{k}^{+}\right) & =\mathbf{h}\left(\mathbf{x}, \mathbf{y}, \mathbf{z}\left(t_{k}^{-}\right)\right)
\end{aligned}
$$

The algebraic equations (1) represent the network equations, whose response is assumed instantaneous under the quasisinusoidal (or phasor) approximation.

The differential equations (2) relate to a wide variety of phenomena and controls ranging from the short-term dynamics of generators, turbines, governors, Automatic Voltage Regulators (AVRs), Static Var Compensators (SVCs), induction motors,

M.-E. Grenier (grenier.marie.eve@hydro.qc.ca) and D. Lefebvre (lefebvre.daniel.4@hydro.ac.ca) are with the TransEnergie division of HydroQuébec, Complexe Desjardins, Tour Est, CP 10000, Montreal H5B 1H7, Canada. Thierry Van Cutsem (t.vancutsem@ulg.ac.be) is with the Belgian National Fund for Scientific Research (FNRS) at the Dept. of Electrical Engineering and Computer Science (Montefiore Institute) of the University of Liège, Sart Tilman B37, B-4000 Liège, Belgium.
HVDC links, etc. to the long-term dynamics of secondary frequency and voltage control, load self-restoration, etc.

The discrete-time equations (3) capture the discrete controls and protections that act on the system, ranging from the fast switching of shunt compensation to long-term controls such as changes in generator setpoints, Load Tap Changers (LTCs), OverExcitation Limiters (OELs), etc. The corresponding (shunt susceptance, transformer ratio, etc.) variables are grouped into $\mathbf{z}$, which undergoes step changes from $\mathbf{z}\left(t_{k}^{-}\right)$to $\mathbf{z}\left(t_{k}^{+}\right)$at some times $t_{k}$.

Full Time Scale (FTS) simulation deals with all three sets of equations. In spite of the availability of variable step size algorithms, this remains in practice a heavy approach in terms of data preparation and output processing, providing too much detail for certain applications, with a high computational effort.

As already mentioned, the QSS approximation consists in decomposing $\mathbf{x}$ into a "fast" component $\mathbf{x}_{1}$ and a "slow" component $\mathbf{x}_{2}$, considering the fast part as infinitely fast and replacing the corresponding differential equations by algebraic equilibrium equations. This yields:

$$
\begin{aligned}
\mathbf{0} & =\mathbf{g}\left(\mathbf{x}_{1}, \mathbf{x}_{2}, \mathbf{y}, \mathbf{z}\right) \\
\mathbf{0} & =\mathbf{f}_{1}\left(\mathbf{x}_{1}, \mathbf{x}_{2}, \mathbf{y}, \mathbf{z}\right) \\
\dot{\mathbf{x}}_{2} & =\mathbf{f}_{2}\left(\mathbf{x}_{1}, \mathbf{x}_{2}, \mathbf{y}, \mathbf{z}\right) \\
\mathbf{z}\left(t_{k}^{+}\right) & =\mathbf{h}\left(\mathbf{x}_{1}, \mathbf{x}_{2}, \mathbf{y}, \mathbf{z}\left(t_{k}^{-}\right)\right)
\end{aligned}
$$

in which $\mathbf{f}$ has been decomposed into $\mathbf{f}_{1}$ and $\mathbf{f}_{2}$, corresponding to the fast and slow parts, respectively.

The QSS technique has been extensively used in long-term voltage stability and security analysis [1], [2]. In this context, frequency has been handled as an algebraic variable, assuming that governors act instantaneously according to the permanent speed droop characteristic.

This paper deals with an extension of the above model, taking into account long-term frequency dynamics. The dynamics of concern consists of synchronous generator rotor oscillations with a period in the order of 25 seconds (frequency of 0.04 $\mathrm{Hz}$ ) following a disturbance of the power balance at system level. Perfect coherency between all generators is assumed, thereby neglecting intermachine electromechanical oscillations hopefully damped out by rotor windings and Power System Stabilisers (PSS) [4]. Frequency then becomes a component of $\mathbf{x}_{2}$, together with the state variables stemming from the turbine and governor models.

The motivation for including frequency dynamics is twofold:

- extend the scope of QSS simulation to the analysis of frequency responses following large disturbances. This 
is obviously of particular interest in isolated systems or in the islands resulting from the splitting of a larger interconnection;

- account for possible interactions between frequency and voltages; indeed, following a disturbance, the frequency oscillations take place over the same time interval as long-term voltage dynamics, and the sensitivity of load to voltage induces frequency changes.

Another contribution of this paper is to report on detailed simulations performed on the Hydro-Québec system, in which both QSS models have been compared to FTS simulation. Since it is interconnected through DC links only, this power system operates at its own frequency; besides, it exhibits the above mentioned interaction between voltage and frequency dynamics.

\section{DERIVATION AND SIMUlation OF QSS MODELS}

\section{A. QSS model without frequency dynamics}

We consider network equations written in terms of active and reactive currents (the latter being preferred to powers for their less nonlinear nature). For an $N$-bus system, there are $2 N$ equations (1) involving $N$ voltage magnitudes and $N$ phase angles, all grouped into $\mathbf{y}$.

Generators, governors and AVRs are represented under the "standard" QSS approximation as follows.

Each synchronous machine is characterized by three variables of the type $\mathbf{x}_{1}$ :

$E_{q}$ the emf proportional to field current

$E_{q}^{s}$ the corresponding emf behind saturated synchronous reactances

$\varphi$ the internal rotor (or load) angle [5].

The active and reactive currents injected by a generator are easily expressed in terms of $E_{q}, E_{q}^{s}, \varphi$ and the terminal voltage $V$, as detailed in the Appendix.

The three variables $E_{q}, E_{q}^{s}, \varphi$ are involved in three algebraic equations of the type (5), relating respectively to:

- machine saturation, taken into account through:

$$
E_{q}-k\left(E_{q}, E_{q}^{s}, \varphi, V\right) E_{q}^{s}=0 \quad k>1
$$

where the $k$ function is also detailed in the Appendix;

- voltage regulation by AVR. This relationship may, for instance, take on the form:

$$
E_{q}-G\left(V^{o}-V\right)=0
$$

where $G$ is the open-loop steady-state gain of the AVR and $V^{o}$ its voltage setpoint;

- speed regulation. Assuming the entire conversion of turbine mechanical power $P^{m}$ into active power $P$ and considering the steady-state governor effect yields:

$$
P-P^{m}=P\left(E_{q}, E_{q}^{s}, \varphi, V\right)-P^{o}+\alpha_{g} \omega=0
$$

where $P^{o}$ is the power setpoint, $\omega$ the per unit frequency (or angular frequency) deviation from nominal value, and $\alpha_{g}$ is a participation factor, function of the permanent speed droop and turbine rating [5].
In turn, the additional $\omega$ variable (common to all generators) is balanced by the phase angle reference equation:

$$
\theta_{r}=0
$$

where $r$ denotes the reference bus. The formulation $(10,11)$ is similar to that of a "distributed slack-bus" or "governor power flow" model (e.g. [6]). When $\mathbf{z}$ changes, so does $\omega$, since it is an algebraic variable.

Thus, for a $g$-machine system, there are $3 g$ variables $E_{q}, E_{q}^{s}, \varphi$ and one variable $\omega$ balanced by $3 g$ equations of the type $(8,9,10)$ and by Eq. (11).

We account for the sensitivity of load power to voltage and frequency through the exponential model:

$$
\begin{aligned}
P & =P^{o}(1+\omega)^{\gamma} \sum_{j=1}^{3} a_{j}\left(\frac{V}{V^{o}}\right)^{\alpha_{j}} \\
Q & =Q^{o}(1+\omega)^{\delta} \sum_{j=1}^{3} b_{j}\left(\frac{V}{V^{o}}\right)^{\beta_{j}}
\end{aligned}
$$

\section{B. QSS model with frequency dynamics}

We now consider the incorporation of frequency dynamics into the previous model. As mentioned in the Introduction, this extension relies on the common (or single) frequency assumption, valid for long-term studies. Under this assumption, the system can be modelled as shown in the bloc diagram of Fig. 1, where, for the $i$-th generator $(i=1, \ldots, g), P_{i}^{m}$ is the mechanical power, $P_{i}$ the active power production, $z_{i}$ the valve opening, and $M_{i}$ the inertia constant of rotating masses. Mechanical damping is neglected.

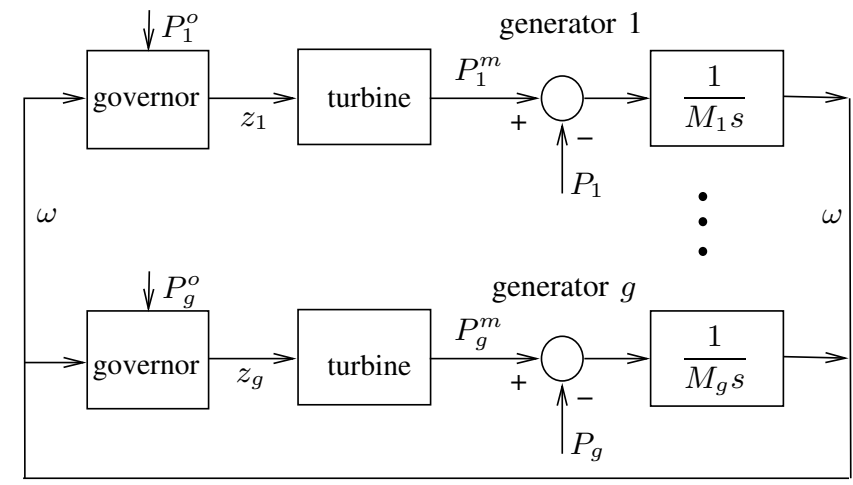

Fig. 1. Common frequency model of the system

One easily derives from Fig. 1:

$$
M_{i} s \omega=P_{i}^{m}-P_{i} \quad i=1, \ldots, g
$$

and by summing over all generators:

$$
M_{T} s \omega=\eta
$$

where $M_{T}=\sum_{i=1}^{g} M_{i}$ is the total inertia and $\eta$ the total power imbalance:

$$
\eta=\sum_{i=1}^{g} P_{i}^{m}-\sum_{i=1}^{g} P_{i}
$$


Combining (14) and (15) straightforwardly gives:

$$
P_{i}^{m}-P_{i}=\frac{M_{i}}{M_{T}} \eta
$$

which shows that the imbalance between the mechanical and electrical powers of the $i$-th machine is a fraction of the total imbalance at system level.

In this QSS model, Eqs. $(8,9)$ relative to the generator and its AVR still hold, while (10) is replaced by (17). The rôle of additional algebraic variable played by $\omega$ in (10) is now played by $\eta$, still balanced by (11). $\omega$ becomes a state variable of the type $\mathbf{x}_{2}$, governed by (15). Hence, when $\mathbf{z}$ changes, so does the time derivative of $\omega$, but not $\omega$ itself.

Both the governor and the turbine models bring new state variables of the type $\mathrm{x}_{2}$, and $P_{i}^{m}$ is a function of those variables. These models are further discussed in Section III.

The load model $(12,13)$ is unchanged.

\section{Numerical integration of the model}

When $\mathbf{z}$ changes from $\mathbf{z}\left(t_{k}^{-}\right)$to $\mathbf{z}\left(t_{k}^{+}\right)$, according to the discrete dynamics (7), so do $\mathbf{x}_{1}$ and $\mathbf{y}$, but not $\mathbf{x}_{2}$. The new values of $\mathbf{x}_{1}$ and $\mathbf{y}$ are obtained by solving $(4,5)$ with the Newton method, which requires to solve a sequence of linear systems:

$$
\mathbf{J}_{\mathbf{y x}}\left[\begin{array}{c}
\Delta \mathbf{y} \\
\Delta \mathbf{x}_{1}
\end{array}\right]=-\left[\begin{array}{c}
\Delta \mathbf{g} \\
\Delta \mathbf{f}_{1}
\end{array}\right]
$$

where $\mathbf{J}_{\mathbf{y x}}$ is the Jacobian matrix of $(4,5)$ with respect to $\mathbf{y}$ and $\mathbf{x}_{1}$.

The differential equations (6) are integrated using the wellknown Trapezoidal rule [11]:

$$
\mathbf{x}_{2}(t+h)=\mathbf{x}_{2}(t)+\frac{h}{2}\left(\dot{\mathbf{x}}_{2}(t)+\dot{\mathbf{x}}_{2}(t+h)\right)
$$

where $h$ is the time step. The following partitioned scheme and functional iterations are used. Starting from a predicted value of $\mathbf{x}_{2}(t+h),(4,5)$ are solved to obtain $\mathbf{y}$ and $\mathbf{x}_{1}$, using the Newton iterations (18). These values are substituted into (6) to obtain $\dot{\mathbf{x}}_{2}(t+h)$, which in turn is introduced in (19) to obtain a corrected value of $\mathbf{x}_{2}(t+h)$. The procedure is repeated until the last change in $\mathbf{x}_{2}$ does not trigger new Newton iterations (18), i.e. until the right hand side of (18) remain smaller than a specified tolerance.

Although functional iterations prevent from increasing $h$ beyond some value, for convergence reasons [11], they have been preferred since they allow to use the same Jacobian $\mathbf{J}_{\mathbf{y x}}$ to both integrate the differential equations and solve the numerous discontinuities (7) of a typical QSS simulation.

When frequency dynamics are not included and the evolution is driven by LTCs and OELs, the QSS model has no differential equation (6) (except those stemming from the timing of the discrete transitions, but these do not require functional iterations). The QSS model is then purely discrete and is solved using (18) only.

\section{Application to HydRo-QuÉBEC System}

\section{A. The Hydro-Québec system}

With its long $735-\mathrm{kV}$ transmission corridors between the hydro generation areas in the North and the main load centers in the South part of the province, and its isolated mode of operation, the Hydro-Québec (H-Q) system is exposed to angle, frequency and voltage stability problems.

Being interconnected with the North-East American system through DC links only, the H-Q system operates at its own frequency. Attention is thus paid to frequency excursions following generation incidents and/or the action of system protection schemes such as generation tripping and (remote, underfrequency or undervoltage) load shedding [7]. Although to a lesser extent, line trippings have an impact on frequency due to the voltage changes they induce near the main load centers, which in turn cause the load active powers to change, through their sensitivity to voltages.

Voltage control and stability is a concern near the load centers of Montréal and Québec city. Long-term voltage stability studies are routinely performed at Hydro-Québec using detailed and QSS simulations. Besides static var compensators and synchronous condensers, the automatic shunt reactor switching devices - named MAIS - play an important role in voltage control [8]. These devices are now available in twenty-two $735-\mathrm{kV}$ substations and control a large part of the total 25,500 Mvar shunt compensation. MAIS devices react to voltage drops but also prevent overvoltages by reconnecting shunt reactors when needed.

The system model includes 846 buses and 132 generators. Long-term dynamics involve 371 LTCs acting at different voltage levels with various delays, 89 MAIS devices, 9 OELs protecting the synchronous condensers located near the main load areas.

\section{B. Governor and turbine modelling}

In the H-Q system, $96 \%$ of the electric power are produced by hydro plants. Hence, only hydro plant governors and turbines are considered in the sequel.

Figures 2 to 4 relate to the main three types of governors found in H-Q power plants, namely the mechanical-hydraulic (Fig. 2), the Woodward (Fig. 3) and the Neyrpic regulator (Fig. 4). The latter is of the PID type. In Woodward and Neyrpic regulators, either the gate opening or the electrical power can be controlled (as indicated by the switch in both figures) but only the power control mode is used in practice. To this purpose, the electrical power $P$ is measured and used as input.

The models shown in these figures are routinely used in FTS simulations. In each diagram, the parameter values are those of a particular plant but are representative of most of them. In the Woodward and Neyrpic models, the gain of the derivative (accelerometer) block has been set to zero, to reflect that this part of the controller has been taken out of service after adverse effects (excitation of local modes of rotor oscillation) were identified [9].

The above models are coupled with the well-known hydro turbine model shown in Fig. 5 [5]. 


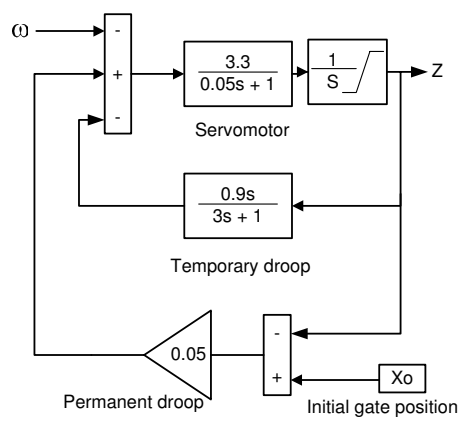

Fig. 2. Mechanical-hydraulic governor: model used in FTS simulation

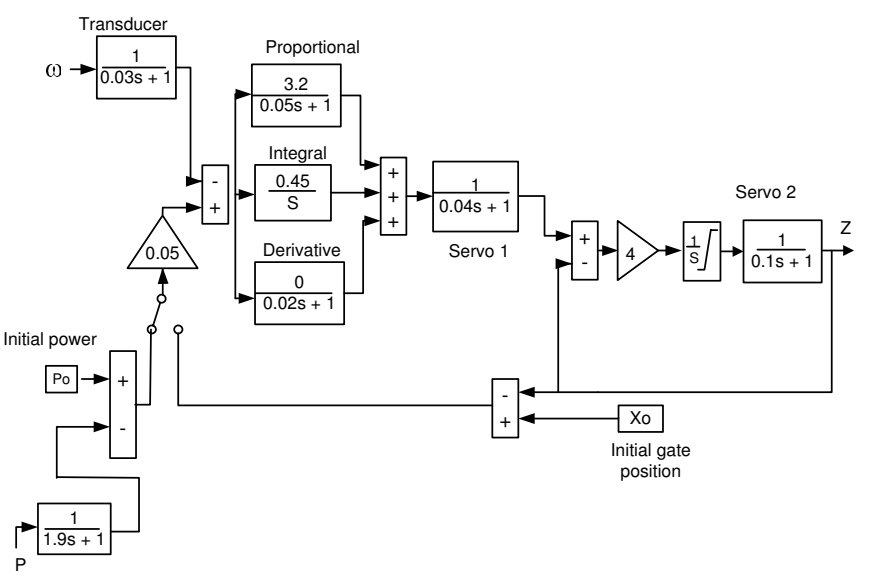

Fig. 3. Woodward governor: model used in FTS simulation

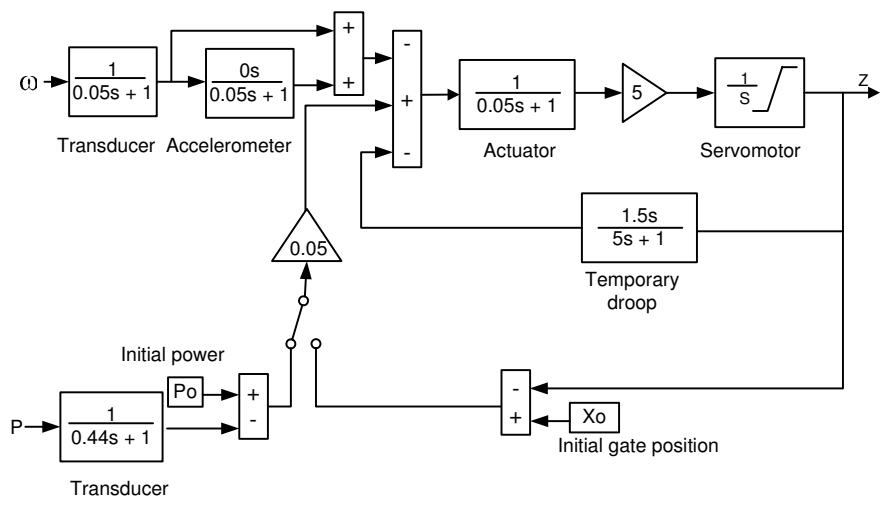

Fig. 4. Neyrpic governor: model used in FTS simulation

$\mathrm{H}$

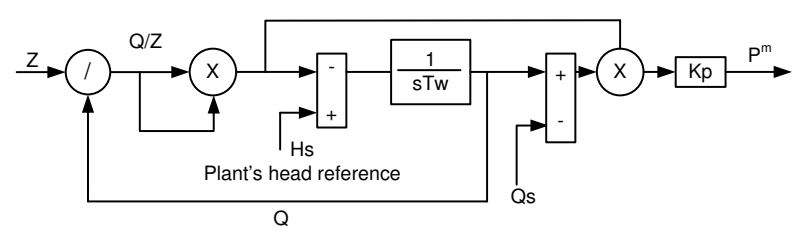

Fig. 5. Hydro turbine: model used in FTS and QSS simulations
To get rid of the fast transients in the QSS model, simplifications have been made as follows. Several disturbances typical of long-term dynamic studies (e.g. generator and line trippings but not short-circuits) have been simulated using the above detailed models, providing the reference frequency and mechanical power evolutions, denoted $\omega_{\text {ref }}(t)$ and $P_{r e f}^{m}(t)$ respectively. Then, the small time constants have been progressively removed. At each step, the simplified governorturbine set has been simulated in an "open-loop" configuration, feeding its input with $\omega_{\text {ref }}(t)$ and collecting the corresponding output $P_{\text {simp }}^{m}(t)$. The models stopped being simplified once the discrepancy $\left|P_{\text {simp }}^{m}(t)-P_{\text {ref }}^{m}(t)\right|$ started being unacceptable. The so obtained simplified models are shown in Figs. 6 to 8, for the three types of governors.

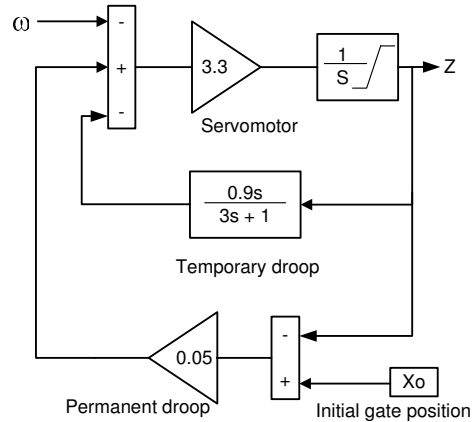

Fig. 6. Mechanical-hydraulic governor: QSS model

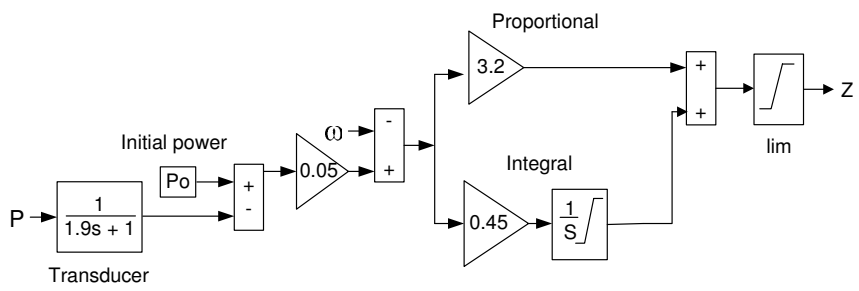

Fig. 7. Woodward governor: QSS model

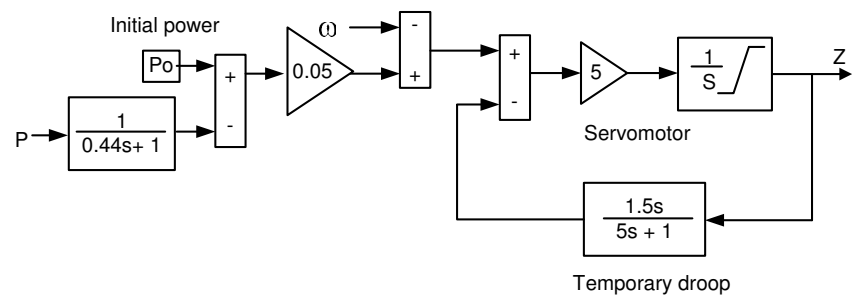

Fig. 8. Neyrpic governor: QSS model

As the water starting times $T_{w}$ of hydro turbines lead to open-loop time constants $0.5 T_{w}$ ranging from 0.4 to $1.6 \mathrm{~s}$, i.e. small compared to the $25 \mathrm{~s}$ period of frequency oscillations, we were tempted to neglect the turbine dynamics as well. However, this simplification led to excessively damped frequency oscillations and, hence, the model of Fig. 5 has been retained. The contribution of the turbine may be explained from its linearized transfer function $\Delta P^{m} / \Delta z=\left(1-s T_{w}\right) /\left(1+s 0.5 T_{w}\right)$, 
which has a non negligible phase angle for $s=j 2 \pi 0.04$. Neglecting the turbine would obviously amounts to neglecting this phase angle.

\section{Simulation of generator trippings}

The examples shown in this section deal with the validation of the QSS model for generator tripping disturbances. As the generators of concern are located in the James Bay area, i.e. far from the load centers, their outage has no impact on load voltages.

Figure 9 shows the frequency evolution following the loss of 350 MW generation, provided by the FTS model and the QSS models with and without frequency dynamics, respectively. The Hydro-Québec ST600 software has been used for FTS simulations.

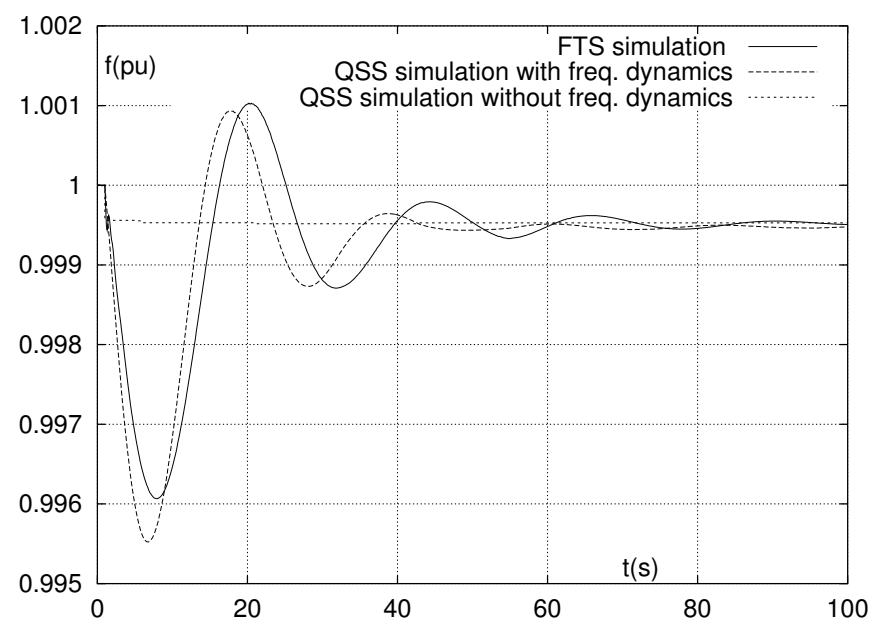

Fig. 9. Loss of $350 \mathrm{MW}$ generation : frequency evolutions

Expectedly, when handled as an algebraic variable, the frequency quickly tends to the equilibrium value reached by the other two models. Compared to FTS simulation, the QSS model with frequency dynamics provides frequency oscillations with a shorter period, a somewhat deeper initial sag and higher damping from the second oscillation. Nevertheless, by and large, the initial impact of the disturbance is well reproduced, the QSS model being somewhat on the safe side.

Figure 10 shows the same evolutions after the tripping of 1050 MW generation. Compared to the previous case, the pseudo-period of oscillations is larger. This is attributable to nonlinear effects, especially over the first oscillation. These nonlinearities originate in particular from turbines that transiently reach their maximum power. In this case also, the QSS model output exhibits a somewhat deeper initial sag while from the second oscillation, the period is shorter and the damping greater. Nevertheless, the QSS response is very satisfactory, especially over the first frequency swing, which is the most important for applications.

The evolution of a $735-\mathrm{kV}$ transmission bus voltage is shown in Fig. 11, for the same disturbance. The initial voltage dip is caused by generator and AVR dynamics, not represented in the QSS model. From there on, the coupling between voltage and frequency can be observed, with voltage oscillations

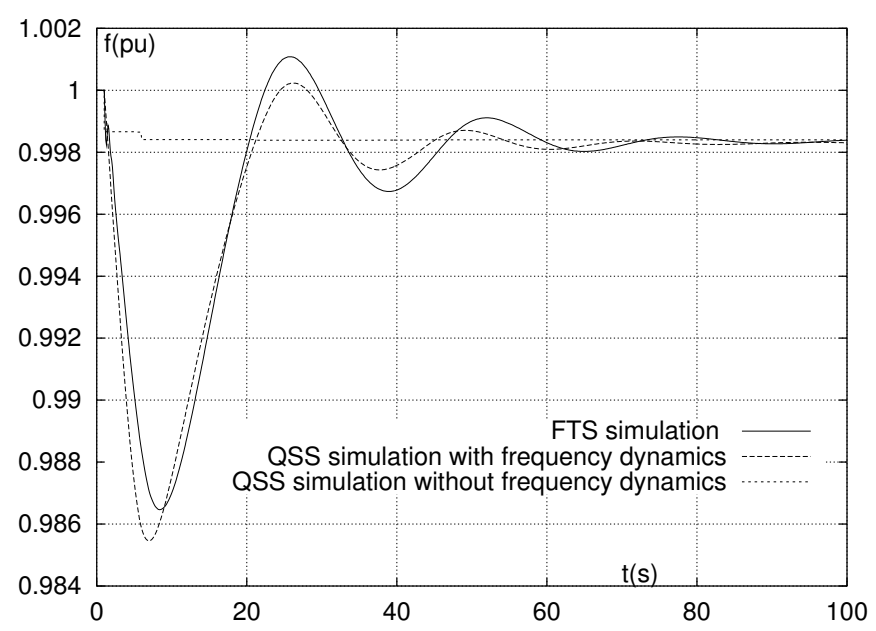

Fig. 10. Loss of $1050 \mathrm{MW}$ generation : frequency evolutions

in opposition to frequency oscillations. The higher damping of QSS oscillations is also seen on this voltage response.

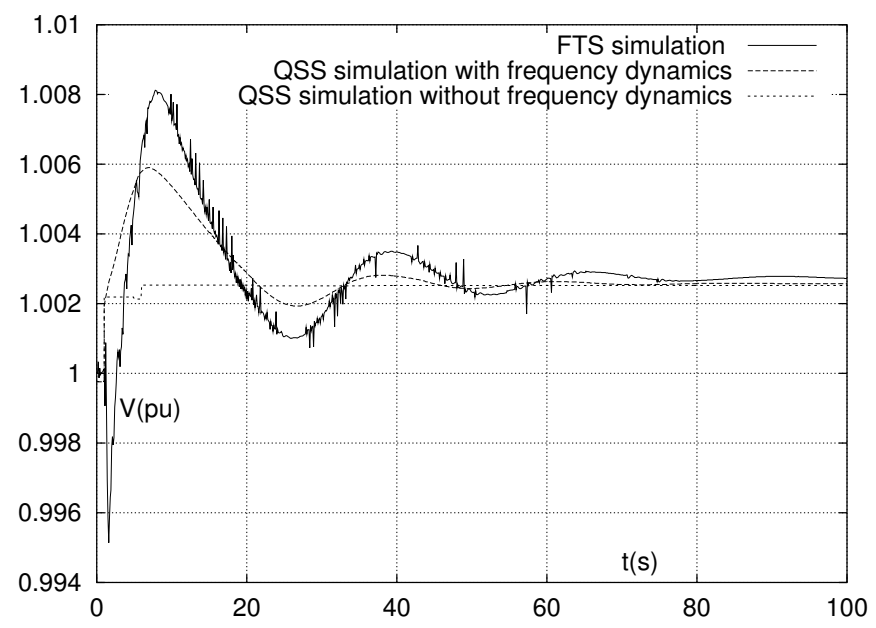

Fig. 11. Loss of $1050 \mathrm{MW}$ generation : voltage evolutions at a $735-\mathrm{kV}$ bus

After carefully checking the QSS model, we came to the conclusion that the algebraic (instantaneous) representation of generators and AVRs (according to Eqs. $(8,9)$ and the ones in the Appendix) was the most likely cause of damping.

To compensate for this approximation, a dynamic corrective term has been added to the static AVR relationship (9) as follows:

$$
E_{q}-G\left(V^{o}-V\right)+\frac{K_{c} s}{1+s T_{c}} \omega=0 \quad K_{c}, T_{c}>0
$$

As can be seen, the added term vanishes in steady state, leaving no permanent error on voltage regulation. The $K_{c}$ and $T_{c}$ parameters have been adjusted in order to match the FTS frequency response in the least square sense.

In the H-Q system, the correction has been found effective when applied to the synchronous condensers in charge of controlling voltages near the major load centers of Montréal and Québec City. This is corroborated by the fact that the new H-Q "multiband" PSS [10] is going to be installed on 
these machines with the objective of damping the frequency oscillation through a smooth, limited in magnitude, modulation of the load voltage. The same $\left(K_{c}, T_{c}\right)$ values have been used on all synchronous condensers, for simplicity.

Figures 12 and 13 show how the frequency and voltage evolutions previously shown in Figs. 10 and 11 are improved by the above correction. The period and the damping of frequency oscillations, as well as the magnitude of the voltage oscillations, are now very close to those found with FTS simulation (at the cost of a little more pronounced initial frequency sag).

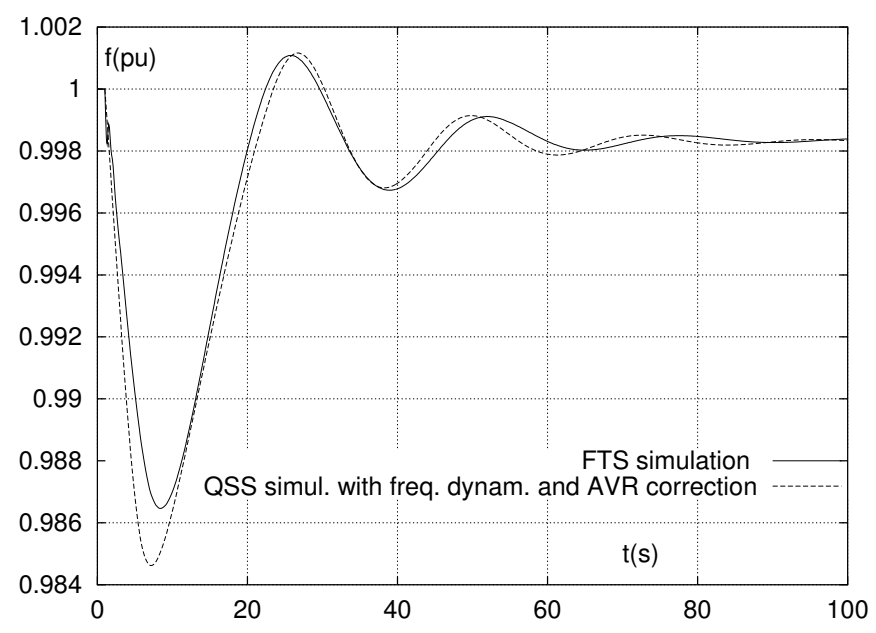

Fig. 12. Loss of $1050 \mathrm{MW}$ generation : frequency evolutions

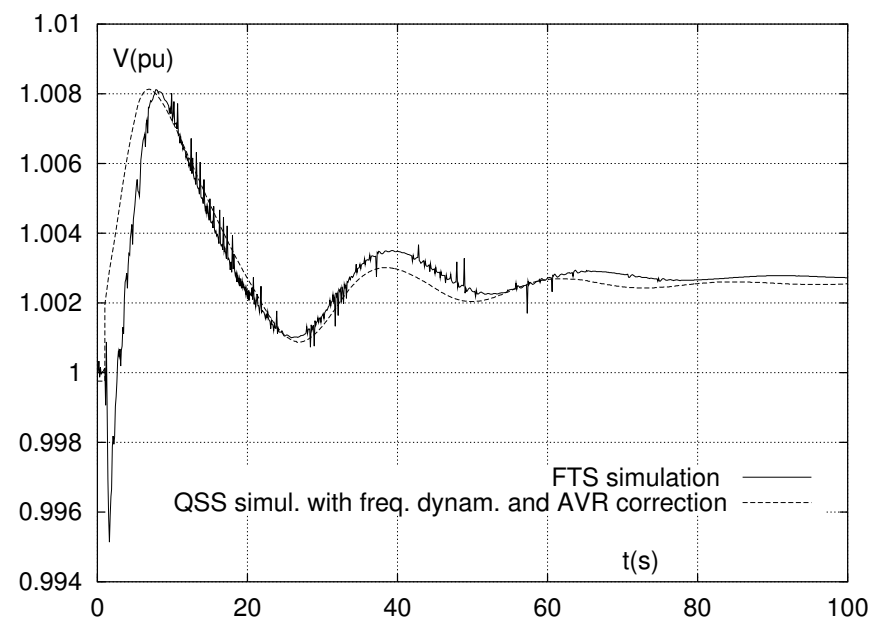

Fig. 13. Loss of $1050 \mathrm{MW}$ generation : voltage evolutions

Of course, further investigations are needed to improve the corrective term. Adding a zero to the transfer function has been found useless. On the other hand, it could be appropriate to limit the amplitude of the correction. Alternatively, a correction of the load model could be envisaged. It must be also ascertained that a single pair of $\left(K_{c}, T_{c}\right)$ values is suitable for all disturbances.

\section{Simulation of line tripping}

The previous section related to generator outages causing significant active power imbalances but relatively small voltage variations. This section, on the contrary, deals with an example of line tripping resulting in significant variations of transmission voltages. It is thus relevant to voltage rather than frequeny stability.

Figure 14 shows the evolution of the voltage at a $735-\mathrm{kV}$ bus in the Montréal area, following the tripping (at $t=1 \mathrm{~s}$ ) of an important $735-\mathrm{kV}$ line. The solid line refers to FTS simulation. On the latter, the voltage jumps at $t=36,93$ and $190 \mathrm{~s}$ correspond to the tripping of shunt reactors by the MAIS devices (see Section III-A).

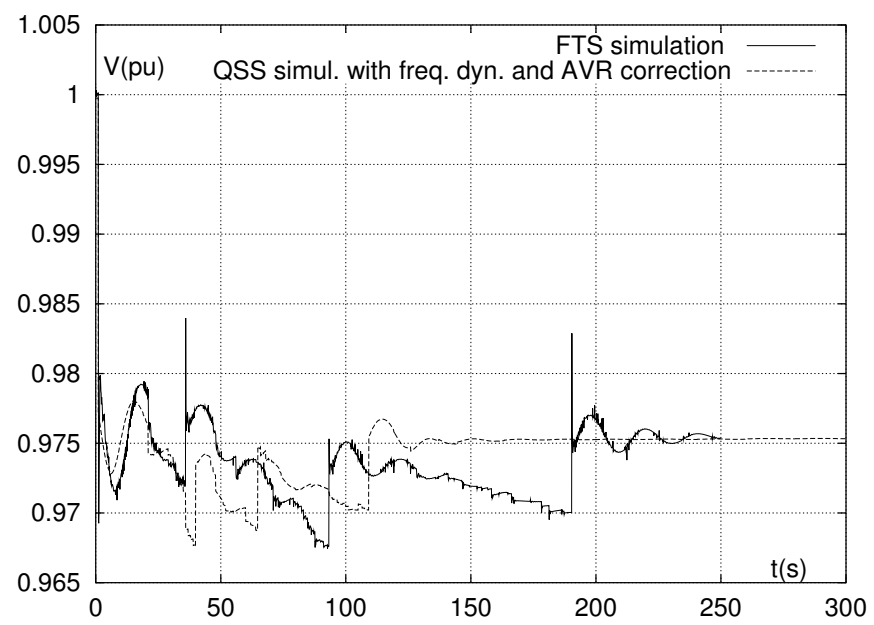

Fig. 14. Tripping of a major 735-kV line : voltage evolutions

The dotted curve is the output of the QSS model including the correction (20). The two responses differ mainly by the times at which shunt reactors are tripped. This difference is due to short-term transients, which are not captured by the QSS model. For instance, in the FTS simulation, the voltage spike at $t=36 \mathrm{~s}$ resets some LTCs (the controlled voltages reentering the deadbands transiently) and delays their reaction. Since the voltage spike is not present in the QSS response, the LTCs move earlier in the latter, which causes the voltage to drop and, hence, the second MAIS to be triggered earlier as well.

In fact, the output of the QSS model is quite acceptable for this $\mathrm{N}-1$ contingency, since it leads to the right number of shunt reactor trippings and the same final voltage. The switching times are not considered critical by $\mathrm{H}-\mathrm{Q}$ engineers and a discrepancy of one shunt reactor would be still acceptable.

Figure 15 offers a zoom on the outputs provided by the three QSS models. As can be seen, including the frequency dynamics has some impact on the switching times. However, when comparing these curves with the FTS one, there is no clear evidence of an improvement in the switching sequence brought by the extension of the QSS model to frequency dynamics. Finally, whether the AVR correction (20) is used or not does not make a significant difference. 


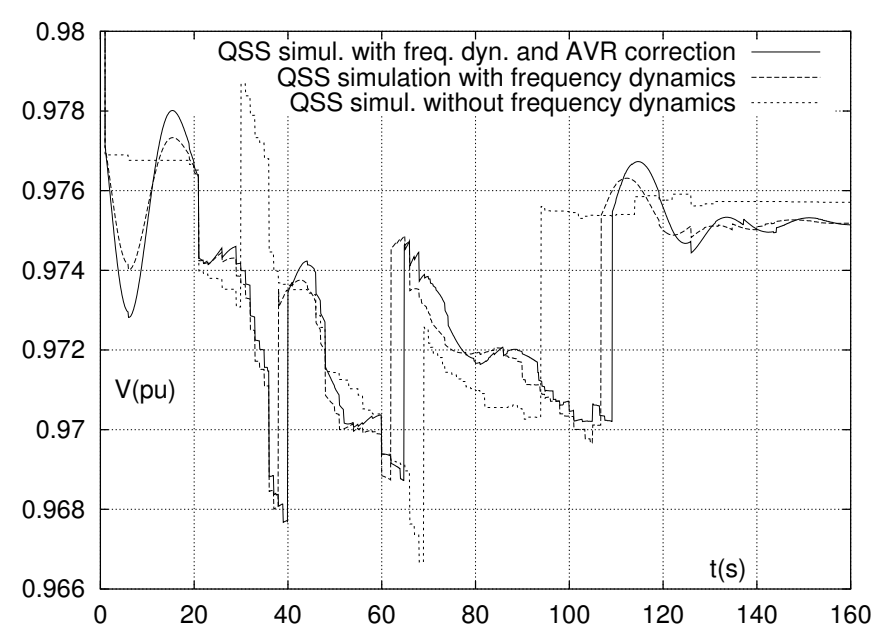

Fig. 15. Tripping of a major 735-kV line : voltage evolutions

\section{E. Computational aspects}

The relatively small time constants introduced by the hydro turbine model led us to select an integration time step of $0.1 \mathrm{~s}$ for the QSS simulation with frequency dynamics. This value guarantees a good convergence of the functional iterations mentioned in Section II-C. It also provides a good accuracy, as indicated by the fact that two simulations with time steps of 0.1 and $0.05 \mathrm{~s}$, respectively, yield indiscernible frequency evolutions.

As mentioned in Section II-C, when frequency dynamics is not included, the QSS model is purely discrete. A step size of $1 \mathrm{~s}$ is used in this case.

The time step of FTS simulation is $0.0083 \mathrm{~s}$ (half cycle at $60 \mathrm{~Hz})$.

Table I give a sample of computing times obtained with an AMD 1.9-GHz PC running Windows 2000. The first case is the one shown in Figs. 12 and 13, leading to a marked frequency swing but few LTC changes and no MAIS action. On the contrary, the last six cases refer to severe doubleline trippings with many LTCs and MAIS responding, but moderate frequency swings. The unstable cases have been obtained by increasing the pre-contingency power transfer in the transmission corridor of the outaged line.

These results show that including the frequency dynamics in the QSS model leads to an increase in computing time by a ratio of 4 to 14 . However, the so improved QSS simulation is still from 60 to 120 times faster than FTS simulation! The very first objective of the QSS approximation is thus fully achieved. Even with the frequency dynamics representation, this technique remains well suited for fast applications.

\section{CONCLUSION}

This paper deals with the extension of a quasi steadystate model previously used for long-term voltage stability analysis, so as to incorporate the frequency dynamics that takes place over the same time range. The model relies on a common frequency assumption, neglecting oscillations between generators.
TABLE I

COMPUTING TIMES (IN SECONDS)

\begin{tabular}{|c|c|c|c|}
\hline \multirow{2}{*}{ case description } & \multicolumn{3}{|c|}{ computing time of } \\
& FTS & \multicolumn{2}{|c|}{ QSS simulation } \\
simulation & \multicolumn{2}{|c|}{$\begin{array}{c}\text { with } \\
\text { without }\end{array}$} \\
\hline $\begin{array}{c}\text { generator tripping far } \\
\text { load center; system stable }\end{array}$ & 237 & 4 & 1 \\
\hline $\begin{array}{c}\text { double-line tripping } \\
\text { system stable; 350 s simulated }\end{array}$ & 893 & 10 & 1 \\
\hline $\begin{array}{c}\text { same but long-term } \\
\text { voltage unstable }\end{array}$ & 895 & 14 & 1 \\
\hline $\begin{array}{c}\text { double-line tripping } \\
\text { system stable; 350 s simulated }\end{array}$ & 954 & 10 & 2 \\
\hline $\begin{array}{c}\text { same but long-term } \\
\text { voltage unstable }\end{array}$ & 1007 & 8 & 2 \\
\hline $\begin{array}{c}\text { double-line tripping } \\
\text { system stable; 300 s simulated }\end{array}$ & 752 & 7 & 1 \\
\hline $\begin{array}{c}\text { same but long-term } \\
\text { voltage unstable }\end{array}$ & 732 & 8 & 1 \\
\hline
\end{tabular}

The paper also reports on tests performed on the HydroQuébec system in order to validate this simplified model with respect to detailed time simulations.

Attention has been paid to the turbine and governor models. The QSS model has been obtained by eliminating fast responding components from the detailed models routinely used in stability studies. It has been found, however, that the hydro turbine response cannot be ignored, in spite of its small time constant.

All in all, the model reproduces very satisfactorily the first frequency swing after a disturbance, although with a little deeper initial sag. The damping of the subsequent oscillations is a bit more pronounced due to presumably the static representation of generators and AVRs. This can be adjusted by adding a dynamic correction term to the AVR relationship.

The initial objective of saving computational effort has been fully met. Indeed, although the step size is 10 times smaller than that of the QSS model without frequency dynamics, the simulation is from 60 to 120 times faster than with the full model.

The voltage dependence of load power has an impact on frequency dynamics, through the system active power balance. However, representing the frequency dynamics only slightly improves the voltage response to incidents near load centers. In the case of the Hydro-Québec system, the QSS simulation does not better reproduce the sequence of shunt reactor trippings when frequency dynamics is added.

Hence, the applications of the new model are more in the field of improved frequency control and stability. Among them, let us quote : secondary frequency control, underfrequency load shedding (or remote load shedding after generation shedding) and the damping of frequency oscillations through dedicated power system stabilizers. Besides, it will be incorporated in the training simulator described in [3].

\section{ACKNOWLEDGMENT}

Marie-Eve Grenier wants to thank Hydro-Québec for giving her the opportunity to work on this project within the context of her DEA degree at the Univ. of Liège. 


\section{REFERENCES}

[1] T. Van Cutsem, C. Vournas, Voltage Stability of Electric Power Systems, Boston, Kluwer Academic Publishers, 1998

[2] T. Van Cutsem, J. Kabouris, G. Christoforidis, C.D. Vournas, "Application of real-time voltage security assessment to the Hellenic interconnected system", IEE Proc. on Generation, Transmission and Distribution, vol. 152, No 1, pp. 123-131, Jan. 2005

[3] T. Van Cutsem, G. Hasse, C. Moors, S. Guillon, R. Mailhot, "A new training simulator for improved voltage control of the HydroQuébec system”, Proc. IEEE Power System Conference and Exhibition (PSC\&E), New York, Oct. 2004

[4] Boeing Computer Services, "Long-term system dynamics simulation methods", EPRI Report EL-3894, Feb. 1985

[5] P. Kundur, Power system stability and control, Mc Graw Hill (EPRI Power System Engineering Series), New York, 1994

[6] M. Lotfalian, R. Schlueter, D. Idizior, P. Rusche, S. Tedeschi, L. Shu, A. Yazdankhah, "Inertial, governor and agc/economic dispatch load flow simulations of loss of generation contingencies", IEEE Trans. on Power Apparatus and Systems, Vol. 104, 1985, pp. 3020-3028

[7] G. Trudel, S. Bernard, G. Scott, "Hydro-Quebec's defense plan against extreme contingencies", IEEE Trans. on Power Systems, Vol. 14, 1999, pp. 958-965

[8] S. Bernard, G. Trudel, G. Scott, "A 735-kV shunt reactors automatic switching system for Hydro-Québec network", IEEE Trans. on Power Systems, Vol. 11, 1996, pp. 2024-2030

[9] I. Kamwa, D. Lefebvre, L. Loud, "Small Signal Analysis of HydroTurbine Governors in Large Interconnected Power Plants", Proc. PES Winter Meeting, Jan 2002, Vol. 2, pp. 1178-1183

[10] R. Grondin, I. Kamwa, G. Trudel, L. Gerin-Lajoie, J. Taborda, "Modeling and closed-loop validation of a new PSS concept, the multi-band PSS”, Proc. IEEE PES General Meeting, Toronto, July 2003, Vol. 3, pp. $1804-1809$

[11] C.W. Gear, Numerical initial value problems in ordinary differential equations, Prentice Hall, 1971

\section{APPENDIX}

With the armature resistance neglected, the active and reactive powers produced by a synchronous generator are given by:

$$
\begin{aligned}
P & =\frac{E_{q}^{s} V}{X_{d}^{s}} \sin \varphi+\frac{V^{2}}{2}\left(\frac{1}{X_{q}^{s}}-\frac{1}{X_{d}^{s}}\right) \sin 2 \varphi \\
Q & =\frac{E_{q}^{s} V}{X_{d}^{s}} \cos \varphi-V^{2}\left(\frac{\sin ^{2} \varphi}{X_{q}^{s}}+\frac{\cos ^{2} \varphi}{X_{d}^{s}}\right)
\end{aligned}
$$

where $X_{d}^{s}$ and $X_{q}^{s}$ is the saturated direct- and quadrature-axis synchronous reactances, respectively [1], [5]. They relate to their unsaturated values $X_{d}$ and $X_{q}$ through:

$$
X_{d}^{s}=X_{l}+\frac{X_{d}-X_{l}}{k} \quad X_{q}^{s}=X_{l}+\frac{X_{q}-X_{l}}{k}
$$

where $X_{l}$ is the leakage reactance and $k$ is the saturation coefficient involved in (8). According to a widely used saturation model:

$$
k=1+m\left(V_{l}\right)^{n} \quad m, n>0
$$

where $V_{l}$ is the magnitude of the voltage behind leakage reactance. The latter is obtained from the generator voltage $\bar{V}$ and current $\bar{I}$ through:

$$
\bar{V}_{l}=\bar{V}+j X_{l} \bar{I}
$$

Replacing into $(21,22) X_{d}^{s}$ and $X_{q}^{s}$ by their expressions (23) and $k$ by the ratio $E_{q} / E_{q}^{s}$, the active and reactive currents are obtained as:

$$
I_{P}=\frac{P}{V}=\frac{E_{q}^{s} E_{q}}{X_{l} E_{q}+\left(X_{d}-X_{l}\right) E_{q}^{s}} \sin \varphi+\frac{V E_{q}}{2}[
$$

$$
\begin{gathered}
\left.\frac{1}{X_{l} E_{q}+\left(X_{q}-X_{l}\right) E_{q}^{s}}-\frac{1}{X_{l} E_{q}+\left(X_{d}-X_{l}\right) E_{q}^{s}}\right] \sin 2 \varphi \\
I_{Q}=\frac{Q}{V}=\frac{E_{q}^{s} E_{q}}{X_{l} E_{q}+\left(X_{d}-X_{l}\right) E_{q}^{s}} \cos \varphi- \\
V E_{q}\left[\frac{\sin ^{2} \varphi}{X_{l} E_{q}+\left(X_{q}-X_{l}\right) E_{q}^{s}}+\frac{\cos ^{2} \varphi}{X_{l} E_{q}+\left(X_{d}-X_{l}\right) E_{q}^{s}}\right]
\end{gathered}
$$

The $k$ coefficient is expressed in terms of the same variables as follows:

$$
k=1+m\left(V_{l}\right)^{n}=1+m\left[\left(V+X_{l} I_{Q}\right)^{2}+\left(X_{l} I_{P}\right)^{2}\right]^{n / 2}
$$

in which $I_{P}$ and $I_{Q}$ have to be replaced by their expressions $(26,27)$.

Marie-Eve Grenier obtained the B.Sc. degree in Electrical Engineering from McGill University, Montréal, in 2002 and the "Diplôme d'Etudes Approfondies" (DEA) degree from the University of Liège, in 2004. Since 2002 she has been working in the planning department of Hydro-Québec, TransEnergie division.

Daniel Lefebvre received his B.Eng. degree in Electrical Engineering from Ecole Polytechnique, Montréal, in 1990. Since then, he has been with Hydro-Québec, TransEnergie Division where he is involved in operations planning for the Main Network. He is a registered professional engineer.

Thierry Van Cutsem graduated in Electrical-Mechanical Engineering from the University of Liège in 1979, where he obtained the Ph.D. degree in 1984 and is now adjunct professor. Since 1980 he has been with the Belgian National Fund for Scientific Research (FNRS), of which he is now a Research Director. His research interests are in power system dynamics, stability, security and real-time control, in particular voltage stability and security. 